\title{
Thick permalloy films for the imaging of spin texture dynamics in perpendicularly magnetized systems
}

\author{
S. Finizio, ${ }^{1, *}$ S. Wintz, ${ }^{1,2}$ D. Bracher, ${ }^{1}$ E. Kirk, ${ }^{1,3}$ A. S. Semisalova, ${ }^{2}$ J. Förster, ${ }^{4}$ K. Zeissler, ${ }^{5}$ T. Weßels, ${ }^{6}$ \\ M. Weigand, ${ }^{4}$ K. Lenz,${ }^{2}$ A. Kleibert, ${ }^{1}$ and J. Raabe ${ }^{1}$ \\ ${ }^{1}$ Paul Scherrer Institut, 5232 Villigen PSI, Switzerland \\ ${ }^{2}$ Institute of Ion Beam Physics and Materials Research, Helmholtz-Zentrum Dresden-Rossendorf, 01328 Dresden, Germany \\ ${ }^{3}$ Department of Materials, Laboratory for Mesoscopic Systems, ETH Zürich, 8093 Zürich, Switzerland \\ ${ }^{4}$ Max-Planck-Institut für Intelligente Systeme, 70569 Stuttgart, Germany \\ ${ }^{5}$ School of Physics and Astronomy, University of Leeds, LS2 9JT Leeds, United Kingdom \\ ${ }^{6}$ Ernst Ruska-Centre for Microscopy and Spectroscopy with Electrons and Peter Grünberg Institute, \\ Forschungszentrum Jülich, 52425 Jülich, Germany
}

(Received 9 May 2018; revised manuscript received 27 July 2018; published 12 September 2018)

\begin{abstract}
We demonstrate that thick permalloy films exhibiting a weak growth-induced perpendicular magnetic anisotropy can be employed as an ideal test system for the investigation of gyration dynamics in topologically trivial and nontrivial magnetic states ranging from an isolated magnetic skyrmion to more complex $n \pi$ spin configurations.
\end{abstract}

DOI: 10.1103/PhysRevB.98.104415

\section{INTRODUCTION}

The investigation of topological spin textures (such as, e.g., the magnetic skyrmion [1-4]) in material systems exhibiting perpendicular magnetic anisotropy (PMA) has recently been the object of increased attention. This is due to the properties arising from their nontrivial topology due to their topological charge where the topological charge is defined according to the following equation [5]:

$$
Q=\frac{1}{4 \pi} \int \mathbf{m} \cdot\left(\frac{\partial \mathbf{m}}{\partial x} \times \frac{\partial \mathbf{m}}{\partial y}\right) d x d y,
$$

$\mathbf{m}$ being the normalized magnetization vector.

Some of the properties influenced by the topological charge include, e.g., the topological Hall effect [1], the skyrmion Hall effect [1-3], and the topological protection of these entities, which provides an energy barrier against annihilation at defects and pinning sites [4,6]. It is also predicted that the topological charge has a considerable influence over the magnetodynamical processes (e.g., gyration dynamics) of these magnetic configurations [7-9], prompting an experimental verification of such predictions. A verification of this kind usually relies on pump-probe magnetic imaging, combining a high spatial and temporal resolution. However, a fundamental requirement for these experiments is that the dynamical processes need to be reproducible over a number of excitation cycles on the order of $10^{6}-10^{10}$. This comes with the requirement that the Gilbert damping of the magnetic material should be low to allow one to excite the gyration modes with moderate excitation signals and that the material should exhibit a low density of pinning sites.

\footnotetext{
*Corresponding author: simone.finizio@psi.ch
}

The PMA materials typically employed for the investigation of magnetodynamical processes in topological magnetic configurations, such as the magnetic skyrmion, usually consist of NM1/FM/NM2 (FM: ferromagnet, NM: nonmagnetic material) multilayer superlattice stacks optimized for a high PMA. Examples of these multilayer superlattices include $\mathrm{Pt} / \mathrm{Co} / \mathrm{Pt}$ [10], Pt/Co/Ir [11], Pt/CoFeB/MgO [2], and $\mathrm{W} / \mathrm{CoFeB} / \mathrm{MgO}$ [12]. However, these multilayer superlattice stacks are usually afflicted by both a relatively high Gilbert damping (e.g., Pt/Co multilayer superlattices optimized for a high PMA show Gilbert dampings on the order of $0.2[10,13]$ ), leading to short-lived dynamics [14], and a high density of pinning sites, which considerably influences the behavior of the magnetic configuration both statically $[11,15]$ and dynamically [14].

In this paper, we propose an alternative approach to the use of multilayer PMA superlattice stacks for the time-resolved investigation of the dynamical processes in perpendicularly magnetized systems. This solution relies on the use of a much simpler material: permalloy [(Py) $\mathrm{Ni}_{x} \mathrm{Fe}_{1-x}$ alloy]. Thin Py films have been, thanks to a combination of a low Gilbert damping and a relatively low density of pinning sites, one of the favorite systems for the investigation of magnetodynamical processes in in-plane magnetized systems. However, if the Py films are grown at thicknesses above a critical value, the presence of a weak growth-induced PMA leads to the stabilization of a wormlike perpendicularly magnetized stripe domain state [16-21]. This is observed within a relatively wide range of stoichiometries for the $\mathrm{Ni}_{x} \mathrm{Fe}_{1-x}$ alloy, suggesting that the origin for this effect is not only due to magnetostrictive effects [17]. Furthermore, if microstructured disks are fabricated out of these thick Py films, magnetic states ranging from isolated magnetic skyrmions to $n \pi$ magnetic configurations (such as, e.g., the $2 \pi$ state [8]) can be reliably stabilized by tailoring the diameter of the disks [19]. 
The presence of such magnetic states in thick Py films has been known for more than 50 years [16-21], but, here, we investigated the dynamical processes (such as, e.g., the gyration dynamics of the magnetic skyrmions stabilized at the center of the disk structures) of the perpendicularly magnetized states stabilized in thick Py films. We furthermore demonstrate in this paper that the advantages that made thin Py films one of the favorite systems for the study of magnetodynamical processes are maintained also for the thick Py films presented here, allowing us to report on a proof-of-principle measurement of the gyrotropic motion of a magnetic bubble domain in a thick Py microstructured disk to demonstrate the feasibility of using thick Py films as a simple and reliable test bed for the investigation of magnetodynamical processes in topological spin textures.

\section{EXPERIMENT}

Microstructured thick Py (with $\mathrm{Ni}_{81} \mathrm{Fe}_{19}$ stoichiometry) disk elements (diameters ranging from $500 \mathrm{~nm}$ to $3 \mu \mathrm{m}$ ) were lithographically patterned on top of 200-nm-thick xray transparent $\mathrm{Si}_{3} \mathrm{~N}_{4}$ membranes and of $p$-doped $\mathrm{Si}(001)$ substrates. A bilayer of methyl methacrylate and of polymethyl methacrylate was spin coated on top of the substrates prior to the lithographical exposure, which was carried out using a Vistec EBPG 5000Plus 100-keV electron-beam writer. The structures were exposed with an exposure dose of $1500 \mu \mathrm{C} \mathrm{cm}^{-2}$ for the $\mathrm{Si}_{3} \mathrm{~N}_{4}$ membranes and of $900 \mu \mathrm{C} \mathrm{cm}^{-2}$ for the Si substrates to account for the different scatterings of the electrons from the different substrates. Following the lithographical exposure, the resist was developed by immersion in a solution of methyl isobutyl ketone and isopropanol (1:3 by volume) for $60 \mathrm{~s}$, followed by immersion in pure isopropanol for $60 \mathrm{~s}$.

The Py films were deposited by thermal evaporation from a commercial $\mathrm{Ni}_{81} \mathrm{Fe}_{19}$ pellet with a growth rate of about $0.5 \mathrm{~nm} \mathrm{~s}^{-1}$ (measured with a quartz crystal balance) using a Balzers BAE 250 evaporator with a base pressure in the $10^{-7}$-mbars range. Prior to the deposition of the $\mathrm{Py}, \mathrm{a} \mathrm{Cr}$ adhesion layer of $10 \mathrm{~nm}$ was thermally evaporated on top of the substrates. After deposition, the Py films were capped with $5 \mathrm{~nm}$ of $\mathrm{Cr}$ to prevent oxidation. The thickness of the Py films was verified by atomic force microscopy on a reference sample. A thickness of $180 \mathrm{~nm}$ for the Py films was selected as a compromise between the necessity to obtain a weak PMA, which requires thick films [17], and the X-ray photon transmission across the Py, necessary for the scanning transmission $\mathrm{X}$-ray microscopy (STXM) imaging, which requires thin films.

After the deposition of the Py films, the parts of the film grown on top of the unexposed resist areas were lifted off by immersion in pure acetone. The quality of the liftedoff structures was verified by optical and scanning electron microscopies.

The magnitude of the Gilbert damping and of the PMA of the continuous Py films was determined by broadband ferromagnetic resonance (FMR) measurements on equivalent reference samples.

The magnetic configuration of the microstructured Py elements was characterized by $\mathrm{x}$-ray photoemission electron microscopy (PEEM) at the Surface Interface Microscopy (SIM) beamline [22] and by STXM at the PolLux (X07DA) end station [23], both at the Swiss Light Source. Magnetic contrast in the resulting images was achieved through the $\mathrm{x}$-ray magnetic circular dichroism (XMCD) effect [24]. The circularly polarized x rays were tuned to the $L_{3}$ absorption edge of Fe.

Thanks to the $16^{\circ}$ incidence angle of the $\mathrm{x}$ rays with respect to the surface of the sample, the XMCD-PEEM imaging experiments allowed for the investigation of the in-plane component of the magnetic domains in both the continuous films and the microstructured elements fabricated on top of the doped $\mathrm{Si}$ substrates. In particular, the in-plane and out-of-plane spin configurations of the thick Py films were determined by acquiring XMCD-PEEM images of the sample under an azimuthal rotation of $0^{\circ}$ and $180^{\circ}$ and subtracting/adding the two images. Further details on the technique are described in Ref. [22]. The XMCD-PEEM images were acquired under no applied static external magnetic fields (referred to as the remnant state). The spatial resolution for the XMCD-PEEM images presented here is on the order of $50-75 \mathrm{~nm}$. Note that due to the surface sensitivity of PEEM imaging [25] only the magnetization configuration at the top surface of the Py films could be investigated with this technique.

To complement the results obtained from XMCD-PEEM imaging, the Py microstructured disks fabricated on top of X-ray transparent $\mathrm{Si}_{3} \mathrm{~N}_{4}$ membranes were investigated by XMCD-STXM imaging. A Fresnel zone plate with an outermost zone width of $25 \mathrm{~nm}$ was employed to focus the circularly polarized $x$ rays. The entrance and exit slits to the monochromator were set in order to achieve a beam spot on the order of $25 \mathrm{~nm}$. Due to the normal incidence of the $\mathrm{x}$-ray beam with respect to the sample surface, only the outof-plane component of the magnetization of the microstructured Py elements could be resolved with XMCD-STXM imaging.

The response of the Py disks to static magnetic fields was determined with quasistatic XMCD-STXM, and timeresolved STXM imaging was employed to image the magnetization dynamics excited by an oscillating out-of-plane magnetic-field gradient in the Py microstructured elements. The time-resolved STXM imaging experiments were performed using circularly polarized photons of only one helicity (circular negative) through the pump-probe technique as described in detail in Ref. [26]. The pump signal consisted of an oscillating out-of-plane magnetic field, generated by injecting an oscillating current across a $\mathrm{Cu} \Omega$-shaped microcoil, generated with a Tektronix AWG7122C arbitrary waveform generator. The microcoil was lithographically defined to be $2-\mu \mathrm{m}$ wide, $200-\mathrm{nm}$ thick, and was fabricated around a micrometer diameter Py disk. To determine the intensity of the out-of-plane magnetic-field gradient generated by the $\Omega$ shaped microcoil, finite element simulations were carried out with the commercial software suite ANSYS. The magnetic field was simulated with a current injected across the $\Omega$-shaped coil of $50 \mathrm{~mA}$, which corresponds to the maximum of the applied current during the experiments. The current transmitted across the coil was determined with a 50- $\Omega$-terminated real-time oscilloscope (Agilent DSO-S 404A). The results of the simulations are shown in Fig. 1. A clear gradient in the out-of-plane component of the magnetic field can be observed in Fig. 1(b). 

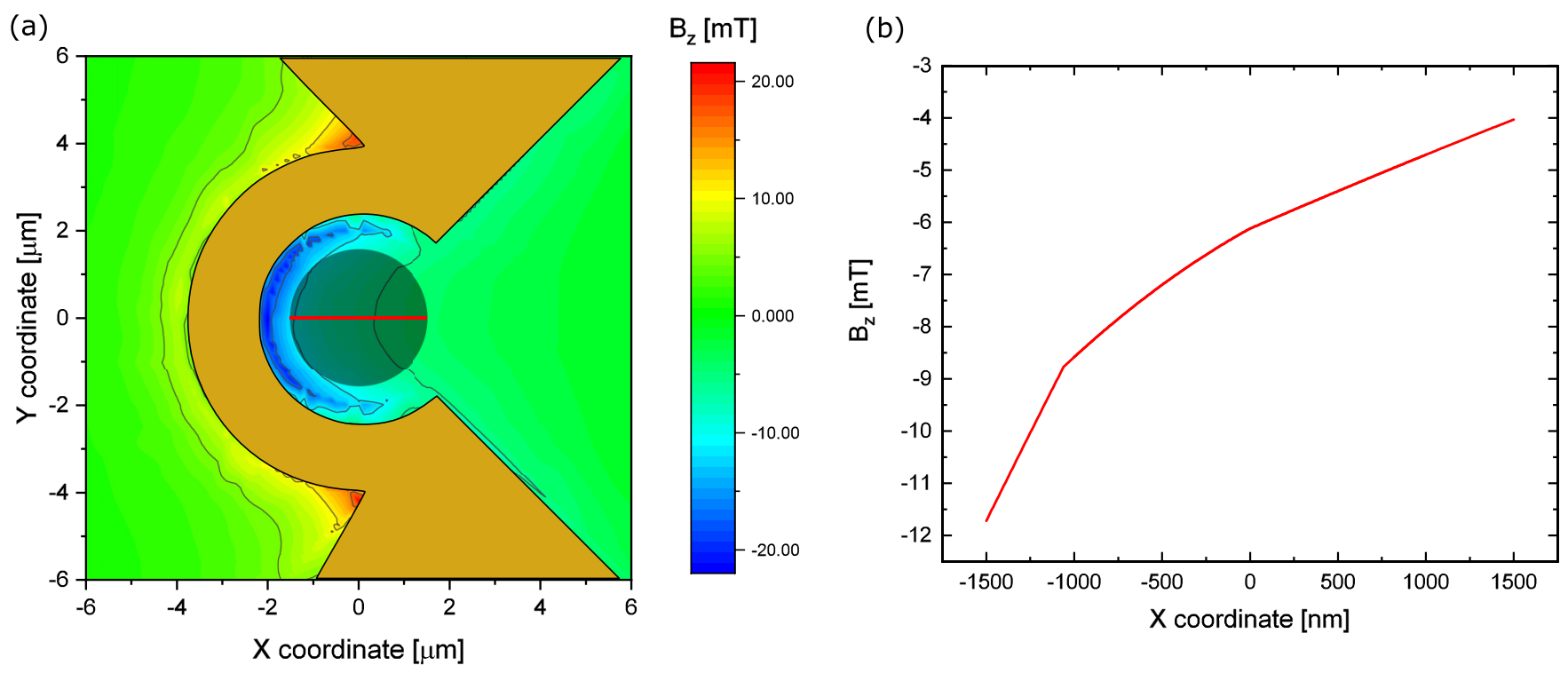

FIG. 1. Finite element simulation of the out-of-plane magnetic field generated by the $\Omega$-shaped microcoil described above. (a) Finite element simulation of the $z$ component of the magnetic field generated by the $\Omega$-shaped microcoil (with a 50-mA current injected across the coil). The circle marks the position of the 3- $\mu$ m disk reported here. (b) Amplitude of the $z$ component of the magnetic field generated by the coil along the red line shown in (a). A clear gradient of the magnetic field across the disk can be observed.

The probing signal is given by the $\mathrm{x}$-ray flashes generated (at a frequency of $500 \mathrm{MHz}$ ) from the synchrotron light source. The wave-form generator is synchronized to the master clock of the synchrotron through a dedicated field programmable gate array setup, which also handles the timing for the acquisition of the time-resolved data. For the data presented in this paper, the time resolution was about 200 ps.

\section{STATIC PROPERTIES}

The thick Py films reported here exhibit a weak growthinduced PMA. The uniaxial anisotropy constant was determined from the value of the effective magnetization $\mu_{0} M_{\text {eff }}$ according to the following relation:

$$
\mu_{0} M_{\mathrm{eff}}=\mu_{0} M_{\mathrm{s}}-2 \frac{K_{\mathrm{u}}}{M_{\mathrm{s}}}
$$

where $M_{\mathrm{s}}=765.8 \mathrm{kA} \mathrm{m}^{-1}$ is the saturation magnetization obtained from superconducting quantum interference device magnetometry. The value of $\mu_{0} M_{\text {eff }}$ was determined from FMR measurements by fitting both the frequency and the polar angular dependencies of the resonance field. The PMA constant was measured to be $29.6 \mathrm{~kJ} \mathrm{~m}^{-3}$.

This weak PMA is attributed to shape anisotropy effects caused by the columnar growth of the Py films [16-18]. The columnar growth of the Py films presented here was qualitatively verified by scanning electron microscopy imaging of the cross section of the as-grown Py films, an example of which is shown in Fig. 2.

As shown in Fig. 3, the continuous 180-nm-thick Py films stabilize a stripe domain pattern with a domain periodicity of about $250 \mathrm{~nm}$. By measuring the in-plane and out-of-plane contrast across a number of different domains [Fig. 3(e)], it is possible to observe the signature of a Néel domain wall at the top surface of the film, similar to the observations (on a different material) reported in Ref. [27]. This observation is in agreement with the expected configuration (from micromagnetic simulations) of the magnetic domain wall for thick materials with a weak PMA, schematically depicted in Fig. 3(f) where it can be observed that the magnetic domain wall resembles a Bloch domain wall at the center of the film and a Néel domain wall (of opposite chiralities) at the top and bottom surfaces of the Py film, also referred to as Néel closure caps [19,28-32]. It is worth noting here that the expected domain-wall configuration for the thick Py films presented here resembles the one simulated for thick multilayer superlattice stacks exhibiting PMA and asymmetric exchange interaction, providing a further similarity between the spin configurations observed in the thick Py films and those observed in multilayered PMA superlattice stacks [33].

As shown in Fig. 4, if microstructured elements with a circular geometry are fabricated, thanks to the contribution of

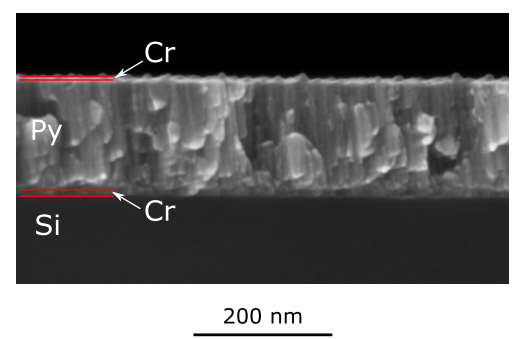

FIG. 2. Cross-sectional scanning electron micrograph of a 180-nm-thick Py film with a 10-nm Cr adhesion layer and a 5-nm $\mathrm{Cr}$ capping layer, showing the columnar growth of Py resulting in the weak PMA observed for these films. 

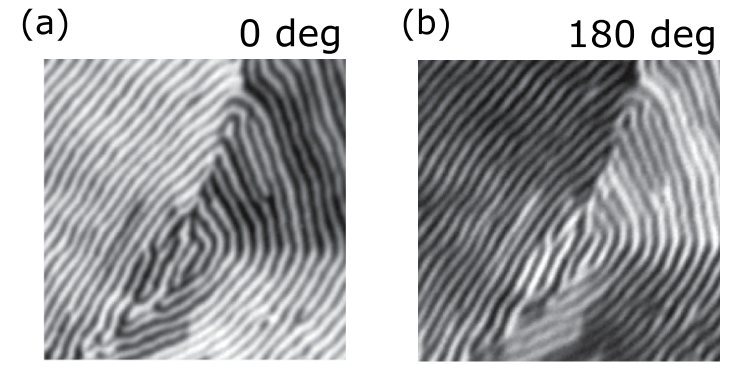

(c)

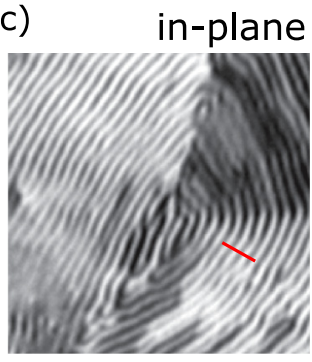

(d) out-of-plane
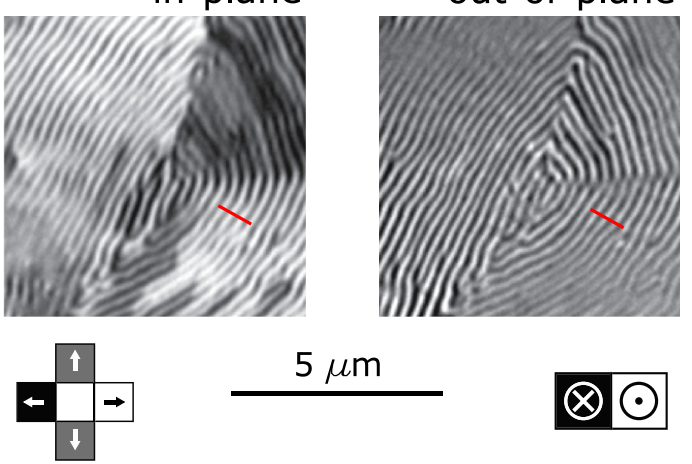

(e)

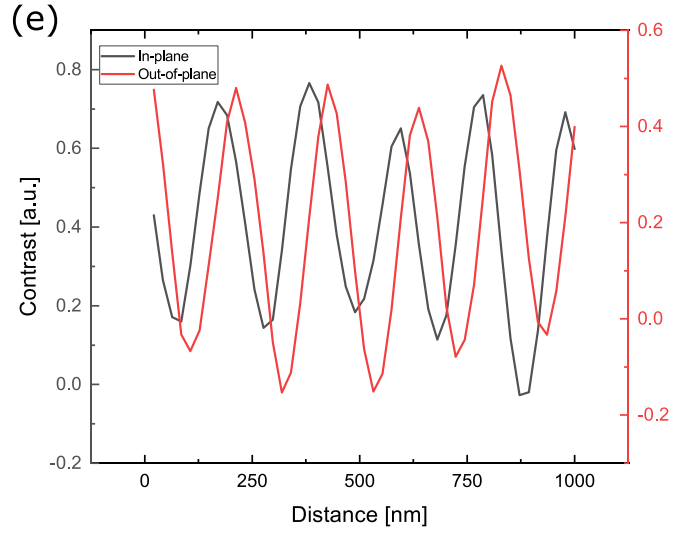

(f)

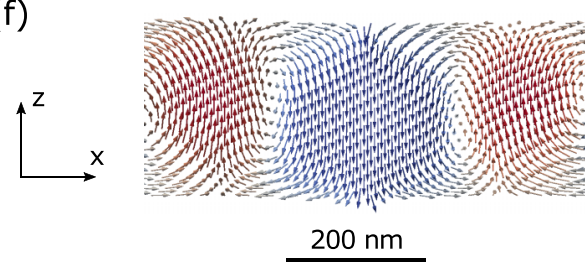

FIG. 3. XMCD-PEEM images of a continuous 180-nm-thick Py film (field of view $7.5 \mu \mathrm{m}$ ), showing (a) and (b) the original XMCDPEEM images employed to determine (c) the in-plane (by subtracting the two images) and (d) the out-of-plane (by summing the two images) magnetic configurations of Py. A stripe domain state with a domain periodicity of about $250 \mathrm{~nm}$ is stabilized. The grayscale arrows in (c) and (d) sketch the direction of the magnetization deduced from the observed XMCD contrast. A line scan across the in-plane and out-of-plane images [marked in red in (c) and (d)] is shown in (e). The characteristic signature of Néel domain walls can be observed [27]. (f) shows an overview of the spin configuration along the thickness of the Py film obtained from micromagnetic simulations.

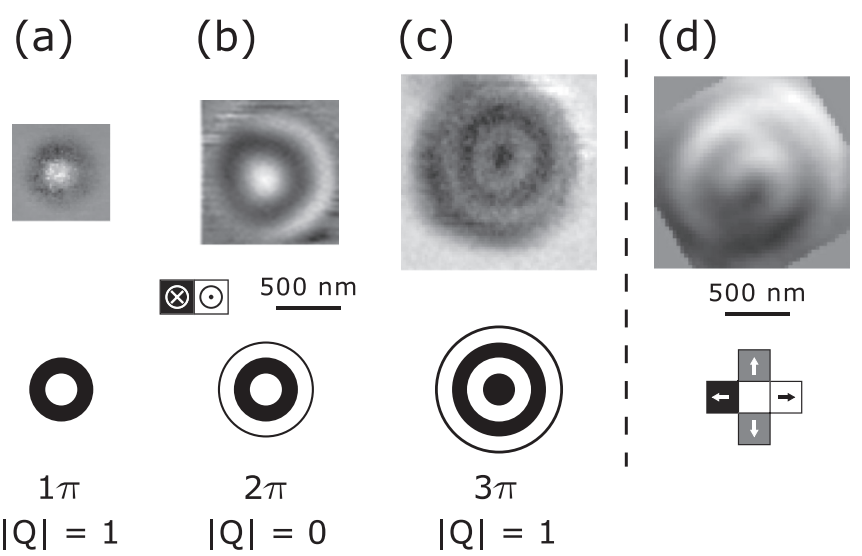

FIG. 4. XMCD-STXM images of (a) a $1 \pi$ state (skyrmion) in a 500-nm-diameter Py disk, (b) a $2 \pi$ state in a 750 -nm-diameter Py disk, and (c) a $3 \pi$ state in a $1-\mu \mathrm{m}$-diameter Py disk. Below each disk, a schematic overview of the out-of-plane magnetic configuration is shown. Image (d) shows a corresponding XMCD-PEEM image of the in-plane component of a 1- $\mu$ m-diameter Py disk where the Néel configuration of the domain walls can be observed. The grayscale arrows indicate the direction of the magnetic contrast.

the shape anisotropy, a magnetic state composed of a central bubble surrounded by concentric ring domains of opposite magnetization will be stabilized [19].

Different magnetic configurations can be attained by tailoring the diameter of the microstructured disk elements. In particular, by selecting an integer multiple $N$ of $250 \mathrm{~nm}$ as the diameter of the microstructured disks, out-of-plane magnetic configurations ranging from an isolated skyrmion $[n=2$, see Fig. 4(a)], corresponding to a topological charge of $|Q|=1$ to a central magnetic bubble surrounded by $n=(N-1)$ ring domains of alternating magnetization $[n \pi$ state-see Figs. 4(b) and 4(c) for the $N=3$ and $N=4$ examples, corresponding, respectively, to topological charges of $|Q|=0$ and $|Q|=1$ ), can be stabilized. Figure 4(d) shows the in-plane component of a Py disk stabilizing a $3 \pi$ state $(N=4)$ where it is possible to observe that the out-of-plane spin texture is coexisting with an in-plane vortex state. This observation is in agreement with previous works [17] where it was observed that the stripe domains align themselves parallel to the direction of this coexisting in-plane magnetic spin texture.

It is worth mentioning here that the magnetic states shown in Fig. 4 were acquired at the remnant state, i.e., in absence of any externally applied magnetic fields. This is in contrast with the majority of the PMA superlattice stacks employed for the stabilization of comparable spin configurations where an out-of-plane magnetic field is necessary [2,11,14,34].

Due to shape anisotropy, the selection of the diameter of the Py disks allows for the reliable and reproducible stabilization of magnetic states with different topological charges, ranging from the isolated skyrmion to the more complex $n \pi$ states. However, as already mentioned above, to study their magnetodynamical properties, a low Gilbert damping and a low density of pinning sites are also desirable requirements. 
(a)

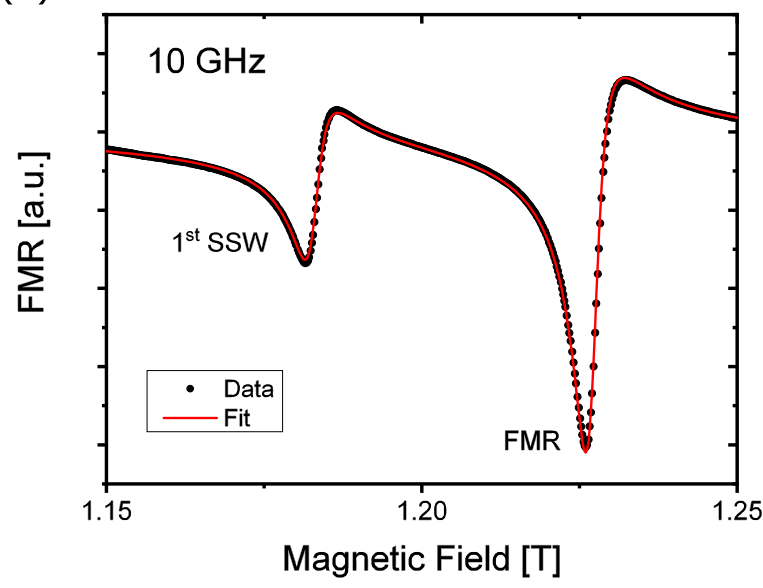

(b)

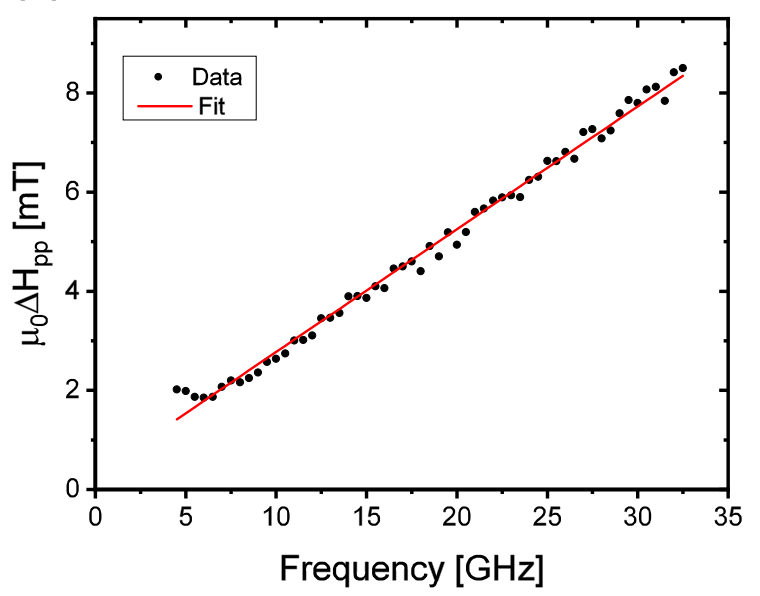

FIG. 5. (a) Microwave absorption spectrum measured at a frequency of $10 \mathrm{GHz}$ with a magnetic field applied along the out-of-plane direction. The fundamental FMR mode and the first standing spin-wave mode are shown. The fit was performed using a complex Lorentzian. (b) Frequency dependence of the peak-to-peak linewidth of the fundamental FMR mode. The slope of the linear fit was used to extract Gilbert damping constant $\alpha$.

The damping of the thick Py films investigated in this work was measured by broadband FMR. The FMR spectra measured under an applied out-of-plane field at a frequency of $10 \mathrm{GHz}$ are shown in Fig. 5(a). A fundamental (uniform) mode and the first standing spin-wave mode can be resolved from the FMR spectra. To allow for the determination of both the resonance field and its linewidth, the data shown in Fig. 5(a) were fitted employing a complex Lorentzian function.

The frequency dependence of the peak-to-peak linewidth $\mu_{0} \Delta H_{\mathrm{pp}}$ is shown in Fig. 5(b), and the value of the Gilbert damping can be extracted using the following relation [35]:

$$
\mu_{0} \Delta H_{\mathrm{pp}}=\frac{2}{\sqrt{3}} \frac{\alpha}{\gamma} \omega
$$

where $\alpha$ is the Gilbert damping constant, $\gamma$ is the gyromagnetic ratio, and $\omega$ is the angular frequency. The value of the Gilbert damping constant was extracted by determining the slope of the frequency-dependent linewidth, and it was found to be about $6.3 \times 10^{-3}$.

To verify that the pinning sites in the Py films described here do not affect the magnetic configuration of the Py microstructured elements, we applied an in-plane magnetic field to a $1-\mu$ m-diameter disk (stabilizing a $3 \pi$ state in the absence of external fields). The application of an in-plane magnetic field causes the displacement of the magnetic bubble domain at the center of the structure due to the influence of the magnetic field on the in-plane vortex state coexisting with the out-of-plane spin texture. In-plane fields of different magnitudes were applied, and a static XMCD-STXM image of the magnetic configuration of the microstructured disk was acquired at each field step. The position of the center of the magnetic bubble domain at each field step, determined from the XMCD-STXM images, is shown in Fig. 6. A smooth displacement of the magnetic bubble with the applied field can be observed for magnetic fields below $20 \mathrm{mT}$, providing an indication that the magnetic bubble domain moves in a low pinning environment. A sharp change in the position of the magnetic bubble domain can be observed for a field of about $\pm 30 \mathrm{mT}$ when approaching from the remnant state. This behavior is, similar to what was observed for the hysteresis loop of magnetic vortices [36], to be attributed to edge repulsion effects.

\section{DYNAMIC PROPERTIES}

In the previous section, we have shown that, by fabricating microstructured disk elements out of thick Py films, it is

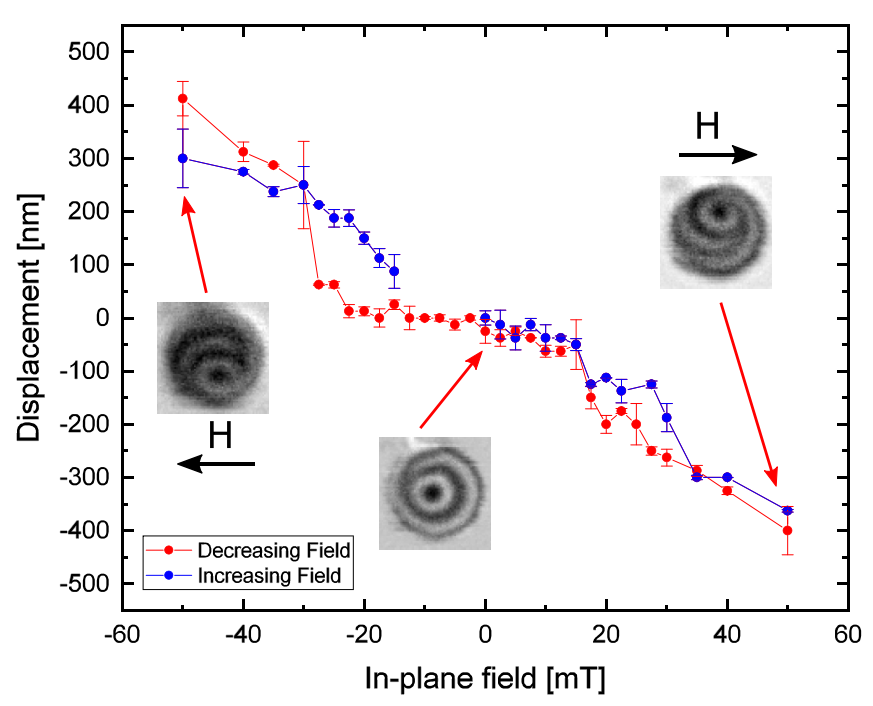

FIG. 6. Position of the center of the central magnetic bubble domain at the center of a $1-\mu \mathrm{m}$-wide-thick Py disk (stabilizing a $3 \pi$ state) as a function of the external in-plane magnetic field (marked by the black arrows in the figure). A relatively smooth motion of the magnetic bubble with the external field can be observed. 


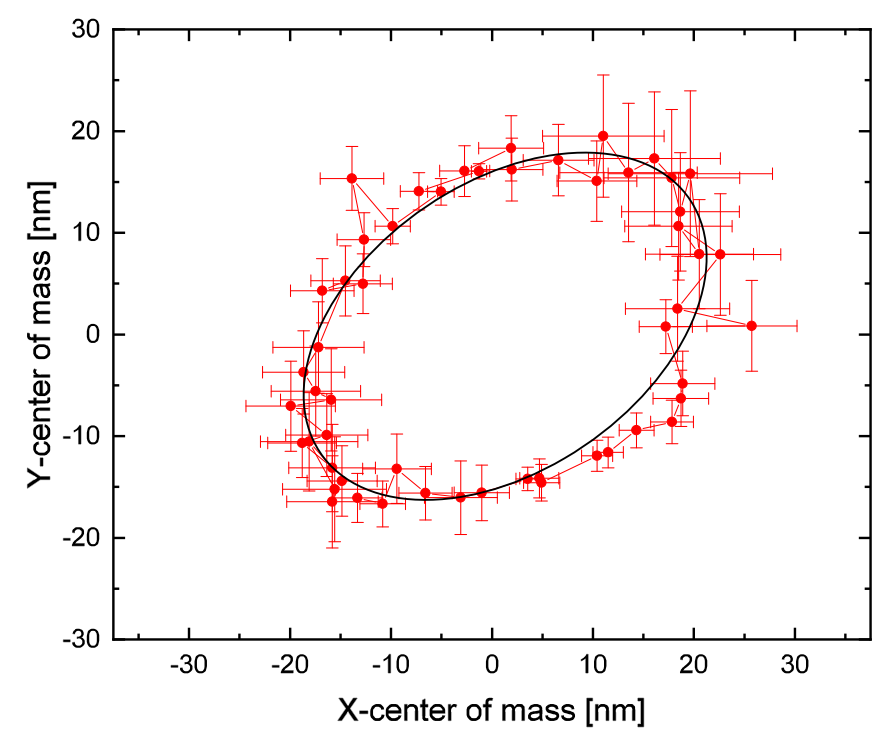

FIG. 7. Position of the center of the magnetic bubble domain at the center of a 3- $\mu$ m-wide-thick Py disk excited with an $85-\mathrm{MHz}$ rf magnetic-field gradient across one cycle of the rf excitation, showing an elliptical orbit. The black line acts as a guide for the eye.

possible to stabilize perpendicularly magnetized magnetic configurations with different topological charges ranging from isolated magnetic skyrmions to more complex $n \pi$ states by proper selection of their diameters. Furthermore, we demonstrated that this material exhibits a low density of pinning sites and a low Gilbert damping. Thick Py films seem therefore to be ideal candidates for the investigation of the dynamical processes of topologically trivial and nontrivial configurations in PMA systems.

To demonstrate the suitability of this material for timeresolved imaging, we conducted a proof-of-principle timeresolved pump-probe measurement. In particular, we investigated the gyrotropic motion of the magnetic domain at the center of a 3- $\mu \mathrm{m}$-wide-thick Py disk. As proposed in the simulations performed in Ref. [7], the gyrotropic motion was excited by generating an oscillating out-of-plane magneticfield gradient, generated by injecting an rf current across an $\Omega$-shaped microcoil.

The proof-of-principle measurement was carried out by injecting rf currents with a frequency of about $85 \mathrm{MHz}$ across the microcoil. The measurements were carried out in the absence of externally applied static magnetic fields (remnant state).

The center of the domain was determined for each frame of the time-resolved image by determining its magnetic center of mass. As shown in Fig. 7, a gyrotropic motion of the magnetic bubble domain stabilized at the center of the Py disk with an elliptical orbit of the semimajor axis of about $15 \mathrm{~nm}$ can be observed in the images (see the Supplemental Material [37]). Due to the requirements of pump-probe imaging, the images shown in Fig. 7 were acquired over $10^{8}-10^{9}$ excitation cycles. This provides a demonstration of the deterministic and reproducible behavior within the limitations of the pump-probe imaging technique of the time-resolved dynamics in the thick Py microstructured elements reported here, giving a final vali- dation that thick Py films exhibiting a weak growth-induced PMA can be employed as an ideal test bed for the study of magnetodynamical processes in perpendicularly magnetized $n \pi$ spin configurations exhibiting different topological charges.

\section{CONCLUSIONS}

In conclusion, we have demonstrated that microstructured disk elements fabricated out of thick Py films grown to achieve a weak PMA stabilize, as observed in a number of previous works [16-21], a perpendicularly magnetized configuration, composed of a circular magnetic domain at the center of the disk surrounded by ring-shaped magnetic domains, the number of which is determined by the ratio between the diameter of the disk and the average width of the stripe domains [19]. Depending on the diameter of the disk structures, perpendicularly magnetized states ranging from an isolated magnetic skyrmion to more complex $n \pi$ states are stabilized. Furthermore, these states are stable in the absence of static out-of-plane magnetic fields.

This material exhibits both a low Gilbert damping and a low density of pinning sites. Therefore, we proposed in this paper to employ this material as an ideal candidate for the investigation of dynamical processes of perpendicularly magnetized systems as it combines the presence of a PMA with the advantages that made Py one of the favorite materials for the study of magnetodynamical processes.

The feasibility of employing thick Py films for the study of dynamical processes in perpendicularly magnetized systems was verified by a proof-of-principle pump-probe imaging experiment where a gyrotropic motion of the magnetic bubble stabilized at the center of a 3- $\mu \mathrm{m}$-wide Py disk was excited by an oscillating out-of-plane magnetic-field gradient.

Finally, the observed weak PMA of the Py films is highly reproducible, even considering films grown in different chambers and growth conditions [17-20], providing a final reason in favor of using this material for the investigation of magnetodynamical processes in perpendicularly magnetized spin configurations.

\section{ACKNOWLEDGMENTS}

This work was performed at the PolLux (X07DA) endstation and at the SIM (X11MA) beamline of the Swiss Light Source, Paul Scherrer Institut, Villigen, Switzerland and at the MAXYMUS end station of the BESSY II light source, Helmholtz-Zentrum Berlin, Berlin, Germany. The research leading to these results has received funding from the European Community's Seventh Framework Programme (Program No. FP7/2007-2013) under Grant Agreement No. 290605 (PSI-FELLOW/COFUND), the European Union's Horizon 2020 Project MAGicSky (Grant No. 665095), and the Swiss Nanoscience Institute (Grant No. P1502). The authors thank J. Lindner for helpful discussions on the FMR results, Y. Yuan for technical support with the superconducting quantum interference device magnetometer, and M. Mruczkiewicz and S. Gliga for helpful discussions on the interpretation of the magnetic configuration of the Py disks. 
[1] N. Nagaosa and Y. Tokura, Nat. Nanotechnol. 8, 899 (2013).

[2] K. Litzius, I. Lemesh, B. Krüger, P. Bassirian, L. Caretta, K. Richter, F. Büttner, K. Sato, O. A. Tretiakov, J. Förster, R. M. Reeve, M. Weigand, I. Bykova, H. Stoll, G. Schütz, G. S. D. Beach, and M. Kläui, Nat. Phys. 13, 170 (2017).

[3] W. Jiang, X. Zhang, G. Yu, W. Zhang, X. Wang, M. B. Jungfleisch, J. E. Pearson, X. Cheng, O. Heinonen, K. Wang, Y. Zhou, A. Hoffmann, and S. G. E. te Velthuis, Nat. Phys. 13, 162 (2017).

[4] A. Fert, V. Cros, and J. Sampaio, Nat. Nanotechnol. 8, 152 (2013).

[5] H. B. Braun, Adv. Phys. 61, 1 (2012).

[6] F. Büttner, I. Lemesh, and G. S. D. Beach, Sci. Rep. 8, 4464 (2018).

[7] C. Moutafis, S. Komineas, and J. A. C. Bland, Phys. Rev. B 79, 224429 (2009).

[8] S. Komineas and N. Papanicolaou, Phys. Rev. B 92, 174405 (2015).

[9] I. Makhfudz, B. Krüger, and O. Tchernyshyov, Phys. Rev. Lett. 109, 217201 (2012).

[10] S. Mizukami, E. P. Sajitha, D. Watanabe, F. Wu, T. Miyazaki, H. Nagauma, M. Oogane, and Y. Ando, Appl. Phys. Lett. 96, 152502 (2010).

[11] K. Zeissler, M. Mruczkiewicz, S. Finizio, J. Raabe, P. M. Shepley, A. V. Sadovnikov, S. A. Nikitov, K. Fallon, S. McFadzean, S. McVitie, T. A. Moore, G. Burnell, and C. H. Marrows, Sci. Rep. 7, 15125 (2017).

[12] S. Jaiswahl, K. Litzius, I. Lemesh, F. Büttner, S. Finizio, J. Raabe, M. Weigand, K. Lee, J. Langer, B. Ocker, G. Jakob, G. S. D. Beach, and M. Kläui, Appl. Phys. Lett. 111, 022409 (2017).

[13] A. Barman, S. Wang, O. Hellwig, A. Berger, E. E. Fullerton, and H. Schmidt, J. Appl. Phys. 101, 09D102 (2007).

[14] F. Büttner, C. Moutafis, M. Schneider, B. Krüger, C. M. Günther, J. Geilhufe, C. v. Korff Schmising, J. Mohanty, B. Pfau, S. Schaffert, A. Bisig, M. Foerster, T. Schulz, C. A. F. Vaz, J. H. Franken, H. J. M. Swagten, M. Kläui, and S. Eisebitt, Nat. Phys. 11, 225 (2015).

[15] I. Gross, W. Akhtar, A. Hrabec, J. Sampaio, L. J. Martinez, S. Chouaieb, B. J. Shields, P. Maletinsky, A. Thiaville, S. Rohart, and V. Jacques, Phys. Rev. Mater. 2, 024406 (2018).

[16] T. Iwata, R. J. Prosen, and B. E. Gran, J. Appl. Phys. 37, 1285 (1966).

[17] N. Saito, H. Fujiwara, and Y. Sugita, J. Phys. Soc. Jpn. 19, 1116 (1964).

[18] D. S. Lo and M. M. Hanson, J. Appl. Phys. 38, 1342 (1967).

[19] P. Eames and E. D. Dahlberg, J. Appl. Phys. 91, 7986 (2002).

[20] J. Ben Youssef, N. Vukadinovic, D. Billet, and M. Labrune, Phys. Rev. B 69, 174402 (2004).
[21] J. Wei, Z. Zhu, H. Feng, J. Du, Q. Liu, and J. Wang, J. Phys. D: Appl. Phys. 48, 465001 (2015).

[22] L. Le Guyader, A. Kleibert, A. Fraile Rodriguez, S. El Moussaoui, A. Balan, M. Buzzi, J. Raabe, and F. Nolting, J. Electron Spectrosc. Relat. Phenom. 185, 371 (2012).

[23] J. Raabe, G. Tzvetkov, U. Flechsig, M. Böge, A. Jaggi, B. Sarafimov, M. G. C. Vernooij, T. Huthwelker, H. Ade, D. Kilcoyne, T. Tyliszczak, R. H. Fink, and C. Quitmann, Rev. Sci. Instrum. 79, 113704 (2008).

[24] G. Schütz, W. Wagner, W. Wilhelm, P. Kienle, R. Zeller, R. Frahm, and G. Materlik, Phys. Rev. Lett. 58, 737 (1987).

[25] G. Schönhense, J. Phys.: Condens. Matter 11, 9517 (1999).

[26] A. Puzic, T. Korhonen, B. Kalantari, J. Raabe, C. Quitmann, P. Jüllig, L. Bommer, D. Goll, G. Schütz, S. Wintz, T. Strache, M. Körner, D. Marko, C. Bunce, and J. Fassbender, Synchrotron Radiat. News 23, 26 (2010).

[27] O. Boulle, J. Vogel, H. Yang, S. Pizzini, D. de Souza Chaves, A. Locatelli, T. O. Mentes, A. Sala, L. D. Buda-Prejabeanu, O. Klein, M. Belmeguenai, Y. Roussigne, A. Stashkevich, S. M. Cherif, L. Aballe, M. Foerster, M. Chshiev, S. Auffret, I. M. Miron, and G. Gaudin, Nat. Nanotechnol. 11, 449 (2016).

[28] C. Moutafis, S. Komineas, C. A. F. Vaz, J. A. C. Bland, and P. Eames, Phys. Rev. B 74, 214406 (2006).

[29] S. Komineas, C. A. F. Vaz, J. A. C. Bland, and N. Papanicolaou, Phys. Rev. B 71, 060405(R) (2005).

[30] M. A. Marioni, N. Pilet, T. V. Ashworth, R. C. O'Handley, and H. J. Hug, Phys. Rev. Lett. 97, 027201 (2006).

[31] J. Wei, H. Feng, Z. Zhu, C. Song, X. Wang, Q. Liu, and J. Wang, J. Magn. Magn. Mater. 432, 245 (2017).

[32] H. A. Dürr, E. Dudzik, S. S. Dhesi, J. B. Goedkoop, G. van der Laan, M. Belakhovsky, C. Mocuta, A. Marty, and Y. Samson, Science 284, 2166 (1999).

[33] W. Legrand, J.-Y. Chauleau, D. Maccariello, N. Reyren, S. Collin, K. Bouzehouane, N. Jaouen, V. Cros, and A. Fert, Sci. Adv. 4, eaat041 (2018).

[34] S. Woo, K. M. Song, X. Zhang, Y. Zhou, M. Ezawa, X. Liu, S. Finizio, J. Raabe, N. J. Lee, S. Kim, S.-Y. Park, Y. Kim, J.-Y. Kim, D. Lee, O. Lee, J. W. Choi, B.-C. Min, H. C. Koo, and J. Chang, Nat. Commun. 9, 959 (2018).

[35] K. Zakeri, J. Lindner, I. Barsukov, R. Meckenstock, M. Farle, U. von Hörsten, H. Wende, K. W, J. Rocker, S. S. Kalarickal, K. Lenz, W. Kuch, K. Baberschke, and Z. Frait, Phys. Rev. B 76, 104416 (2007).

[36] R. P. Cowburn, D. K. Koltsov, A. O. Adeyeye, M. E. Welland, and D. M. Tricker, Phys. Rev. Lett. 83, 1042 (1999).

[37] See Supplemental Material at http://link.aps.org/supplemental/ 10.1103/PhysRevB.98.104415 for a video of the time-resolved XMCD-STXM image. 